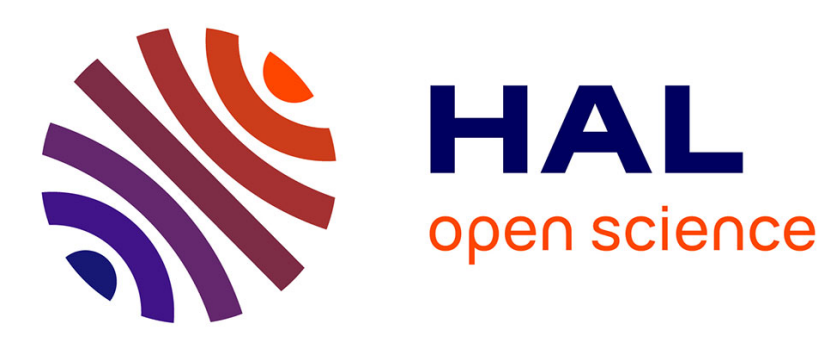

\title{
Mécanisme de formation de la couche diffuse à l'entrée d'une conduite de section circulaire
}

\author{
G. Touchard, P. Humeau, J. Borzeix, H. Romat
}

\section{To cite this version:}

G. Touchard, P. Humeau, J. Borzeix, H. Romat. Mécanisme de formation de la couche diffuse à l'entrée d'une conduite de section circulaire. Revue de Physique Appliquée, 1987, 22 (9), pp.10651069. 10.1051/rphysap:019870022090106500 . jpa-00245644

\section{HAL Id: jpa-00245644 https://hal.science/jpa-00245644}

Submitted on 1 Jan 1987

HAL is a multi-disciplinary open access archive for the deposit and dissemination of scientific research documents, whether they are published or not. The documents may come from teaching and research institutions in France or abroad, or from public or private research centers.
L'archive ouverte pluridisciplinaire HAL, est destinée au dépôt et à la diffusion de documents scientifiques de niveau recherche, publiés ou non, émanant des établissements d'enseignement et de recherche français ou étrangers, des laboratoires publics ou privés. 


\title{
Mécanisme de formation de la couche diffuse à l'entrée d'une conduite de section circulaire
}

\author{
G. Touchard, P. Humeau, J. Borzeix et H. Romat \\ L.E.A.-U.A. 191 du C.N.R.S., Laboratoire de Physique et Mécanique des Fluides, \\ 40, avenue du Recteur Pineau, 86022 Poitiers, France
}

(Reçu le 25 novembre 1986, révisé le 5 février 1987, accepté le 5 mars 1987)

\begin{abstract}
Résumé. - Dans cette étude les phénomènes de formation de la couche diffuse sont analysés à partir de la détermination de la charge convectée dans la couche diffuse s'établissant à l'entrée d'un tube. Le profil des vitesses est déterminé théoriquement ainsi que la charge convectée. Les expériences réalisées pour plusieurs longueurs et diamètres de tubes sont ensuite présentées. Enfin, l'étude des résultats expérimentaux obtenus grâce à l'analyse théorique élaborée nous montre que le courant de paroi est très important dans la zone d'entrée du tube.
\end{abstract}

\begin{abstract}
In this paper we analyse the processus of diffuse layer formation through the phenomenon of charge convection in a diffuse layer appearing at the entrance of a pipe. The velocity profile and the charge convected is computed. Then the experiments obtained for different diameters and lengths of tube are presented. At least, the analyses of the experimental data through the theory elaborated point out the importance of the wall current at the entry of the tube.
\end{abstract}

\section{Introduction.}

Les mécanismes de formation de la double couche électrique apparaissant au contact entre un métal et un liquide, restent encore assez mal connus et dépendent d'un grand nombre de paramètres, notamment de la réaction électrochimique à l'interface [1]. Un des moyens d'investigation, pour mieux connaître ce phénomène, est basé sur le balayage de cette double couche par l'écoulement du liquide. De nombreux travaux ont été faits dans ce domaine et nous ont permis d'analyser le phénomène de transport de charge en fonction du type d'écoulement, du nombre de Reynolds, de la longueur et du diamètre du tube, de la composition et de la température du liquide, et de la nature du matériau des conduites $[2,3]$. Les résultats, en fonction de la longueur des tubes, ont été obtenus pour des tubes de $4 \mathrm{~cm}$ à $4 \mathrm{~m}$ de long [4], mais le phénomène dans la zone d'entrée hydrodynamique n'avait pas été examiné, c'est pourquoi nous avons entrepris cette recherche sur la charge générée au sein d'orifices.

2. Forme analytique du profil de vitesse dans la zone d'entrée d'un tube pour un écoulement laminaire.

De nombreux travaux ont été faits dans ce domaine de la Mécanique des Fluides, nous pouvons citer par exemple ceux de Sparrow, Lin et Lundgren [5], de Gerald, Aiello, Trefil [6], et de Tao et Gupta [7]. La plupart des auteurs reprennent d'ailleurs l'étude de Sparrow en tentant de réduire le nombre de termes à calculer. En 1980, Romat [8], au sein du laboratoire de Physique et Mécanique des Fluides, s'appuyant sur la méthode de Sparrow mais utilisant des méthodes numériques de calcul pour déterminer les différentes grandeurs, nous propose une solution plus exacte à ce problème. Dans cette présente étude, nous nous sommes appuyé sur son analyse pour calculer la vitesse longitudinale, dans le cas d'un orifice circulaire.

Le rapport $\omega$ de la vitesse longitudinale $u$ dans la zone d'entrée d'un tube sur la vitesse moyenne de débit $U$, est fonction de deux coordonnées réduites : $\eta=r / R a$ ( $r$ étant la coordonnée radiale et $R a$ le rayon du tube) et $X^{*} ; X^{*}$ dépend à la fois du nombre de Reynolds $(\operatorname{Re}=2 U R a / \nu)$, de la distance à l'entrée du tube $z$ et du rayon $R a$.

Afin d'exprimer $X^{*}$, il est commode d'introduire une nouvelle variable $X$ proportionnelle à $z$ :

$$
X=2 z /(\operatorname{RaRe}) \text {. }
$$

La coordonnée $X^{*}$ étant liée à $X$ par la relation :

$$
\mathrm{d} X=\varepsilon \mathrm{d} X^{*} \text {. }
$$


$\varepsilon$ étant elle-même une fonction de $X^{*}$ uniquement (Fig. 1) définit comme suit :

$$
\varepsilon=\frac{\int_{0}^{1}\left(2 \omega-1,5 \omega^{2}\right)\left(\partial \omega / \partial X^{*}\right) \eta \mathrm{d} \eta}{(\partial \omega / \partial \eta)_{\eta=1}+\int_{0}^{1}(\partial \omega / \partial \eta)^{2} \eta \mathrm{d} \eta}
$$

avec

$$
\begin{aligned}
& \omega\left(\eta, X^{*}\right) \frac{u\left(\eta, X^{*}\right)}{U}=2\left(1-\eta^{2}\right)+ \\
&+\sum_{i=1}^{\infty}\left(\frac{4}{\alpha_{i}^{2}}\left\{\frac{J_{0}\left(\alpha_{i} \eta\right)}{J_{0}\left(\alpha_{i}\right)}-1\right\} \mathrm{e}^{-\alpha_{i}^{2} X^{*}}\right) .
\end{aligned}
$$

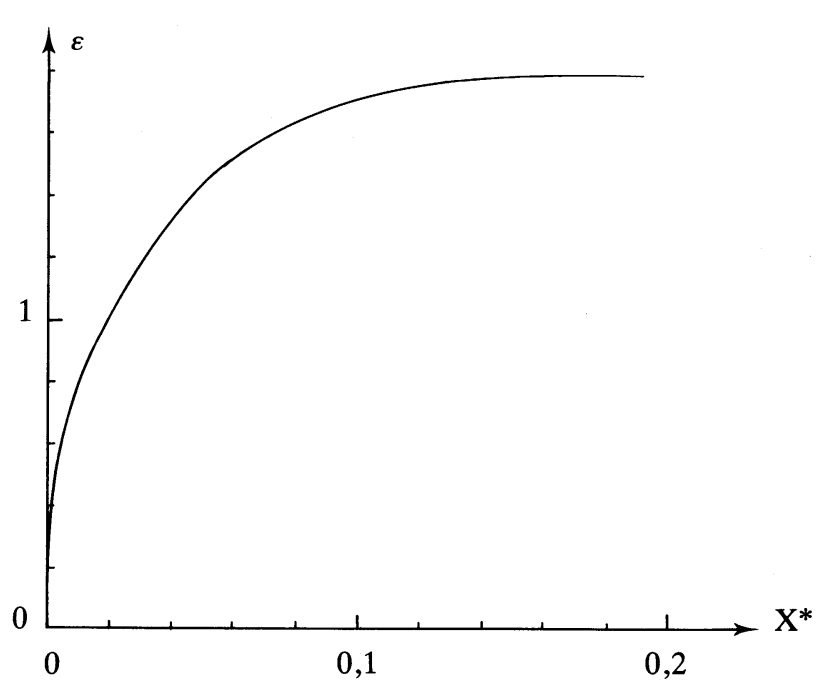

Fig. 1. - Evolution de $\varepsilon$ en fonction de $X^{*}$.

[Variation of $\varepsilon$ in terms of $X^{*}$.]

$J_{0}$ est la fonction de Bessel à l'ordre $0, \alpha_{i}$ sont les zéros de la fonction suivante :

$$
J(\alpha)=J_{1}(\alpha) / J_{0}(\alpha)-0,5 \alpha .
$$

$J_{1}$ étant la fonction de Bessel à l'ordre 1.

Le profil des vitesses étant ainsi défini par la relation (4) en fonction de $\eta$ et de $X^{*}$, il est obtenu en fonction de $r, z, R a$ et $R e$ grâce à la relation entre $X$ et $X^{*}$ (Fig. 2).

Nous avons mis au point un programme de calcul (exécuté sur les ordinateurs du C.I.R.C.E. à Orsay) pour déterminer la vitesse que nous obtenons avec une grande précision en tenant compte notamment d'un très grand nombre de zéros de la fonction $J(\alpha)$ (les valeurs obtenues par Sparrow avaient parfois $40 \%$ d'erreur).

Nous voyons (Fig. 3) une représentation du profil de vitesse $(u / U)$ en fonction de $r / R a$ et pour différentes valeurs de $X$. Ces valeurs de $X$ qui correspondent aux trois valeurs de $X^{*}: 0,02$; 0,$005 ; 0,001$ ont été choisies en fonction de notre

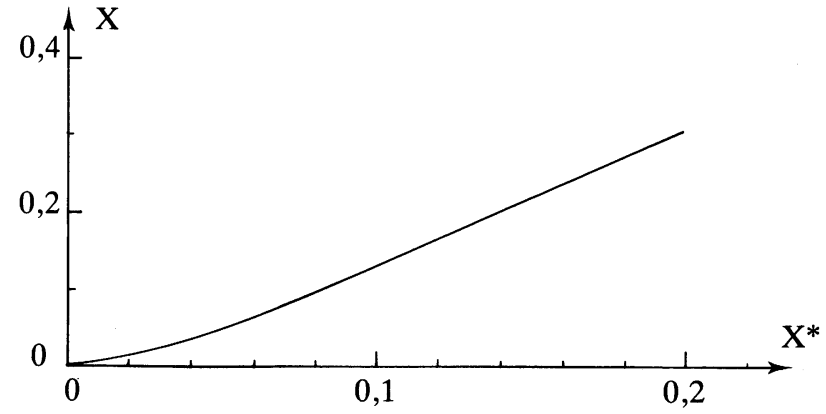

Fig. 2. - Evolution de $X$ en fonction de $X^{*}$.

[Variation of $X$ in terms of $X^{*}$.]

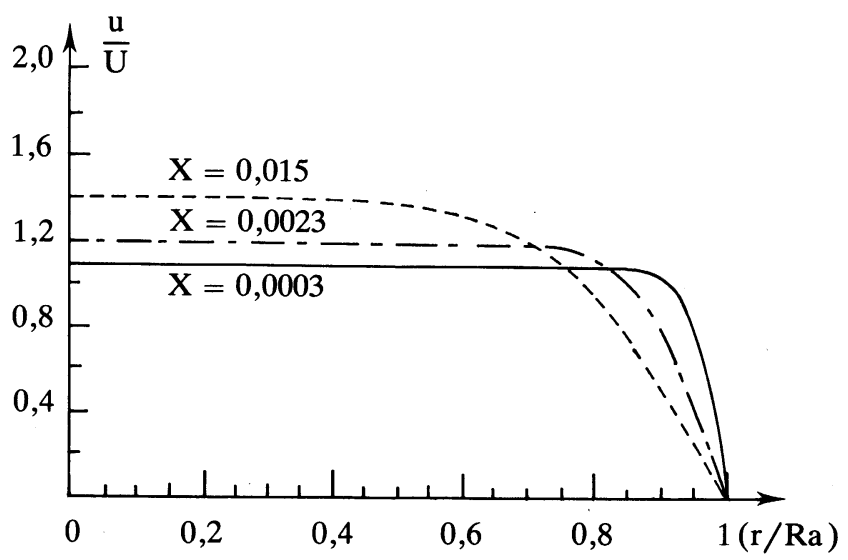

Fig. 3. - Profil des vitesses pour différentes valeurs de $X$. [Velocity profile for differents values of $X$.]

domaine expérimental $(0,5 \mathrm{~mm}<R a<1,5 \mathrm{~mm}$; $1 \mathrm{~mm}<z<5 \mathrm{~mm}$ et $R e<3000)$.

En fonction de ces résultats, nous considérons en première approximation le profil des vitesses dans un orifice circulaire définit comme suit :

- pour

$$
\begin{aligned}
& r \leqq R_{1} \quad u / U=U_{1} \\
& \text { - pour } \\
& r>R_{1} \quad u / U=A(R a-r) . \\
& U_{1}=A\left(R a-R_{1}\right) \\
& \int_{0}^{R a} 2 \pi(u / U) r \mathrm{~d} r=\pi R a^{2} .
\end{aligned}
$$

$U_{1}$ étant déterminé grâce à l'analyse théorique précédente, $R_{1}$ et $A$ sont alors solutions des équa-

3. Densité volumique de charge transportée par un écoulement laminaire dans un orifice cylindrique circulaire.

Nous savons que la densité volumique de charge transportée dans une conduite de façon générale [9], est définie par l'intégrale : 


$$
Q_{1}=\frac{\int_{0}^{R} 2 \pi \rho u r \mathrm{~d} r}{\pi R^{2} U} .
$$

Par ailleurs, s'appuyant sur une étude précédente [10], nous supposerons que les mécanismes de formation de la couche diffuse sont contrôlés par le courant de paroi et non par la diffusion. La couche diffuse se développe alors globalement et son profil est semblable à une couche diffuse établie dont la valeur de la densité volumique de charge à la paroi est fonction de la distance à l'entré $\left(\rho_{\mathrm{P}}=\right.$ $\left.\rho_{\infty}(1-\exp (-b z))\right), b$ étant un coefficient inversement proportionnel au nombre de Reynolds.
En prenant l'expression de la densité volumique de charge adimensionnelle obtenue dans le cas des faibles densités [11], nous aurons :

$$
\rho_{+}=\frac{\rho_{\mathrm{P}^{+}}}{I_{0}\left(\alpha R a_{+}\right)} I_{0}\left(\alpha r_{+}\right)
$$

Les quantités indicées + étant des quantités adimensionnelles, $\alpha$ un coefficient lié aux coefficients de diffusion des ions, et les fonctions $I_{n}$ sont des fonctions de Bessel modifiées d'ordre $n$.

La densité volumique de charge adimensionnelle convectée peut alors être calculée :

$$
\begin{aligned}
Q_{1^{+}}=\frac{2 \rho_{\mathrm{P}^{+}} U_{1}}{\alpha^{2}\left(R a_{+}-R_{1_{+}}\right) R a_{+}^{2} I_{0}\left(\alpha R a_{+}\right)} & {\left[R a_{+} I_{0}\left(\alpha R a_{+}\right)-R_{1_{+}} I_{0}\left(\alpha R_{1_{+}}\right)-\right.} \\
- & \left.\frac{1}{\alpha}\left(\sum_{k=0}^{\infty}(-1)^{k} I_{2 k+1}\left(\alpha R a_{+}\right)-\sum_{k=0}^{\infty}(-1)^{k} I_{2 k+1}\left(\alpha R_{1_{+}}\right)\right)\right]
\end{aligned}
$$

Les valeurs de $Q_{1^{+}}$obtenues pour des cas particuliers correspondant notre domaine expérimental sont reproduites figure 4.

Nous voyons que la densité volumique de charge convectée passe généralement par un maximum. Pourtant la charge totale dans une couche diffuse en formation est croissante avec le temps de contact entre le liquide et le solide. Aussi, si le profil des vitesses était réellement plat, quel que soit le nombre de Reynolds, lorsque celui-ci croît, puisque la vitesse croît et donc le temps de contact entre la paroi de l'orifice et le liquide diminue, on devrait avoir une diminution constante de la charge convectée. En fait, particulièrement pour les petits diamètres, nous avons vu, figure 3, que l'hypothèse des profils plats est très approximative. Pour des diamètres plus élevés, le profil des vitesses tend vraisemblablement mieux vers un profil plat, mais par contre le rapport
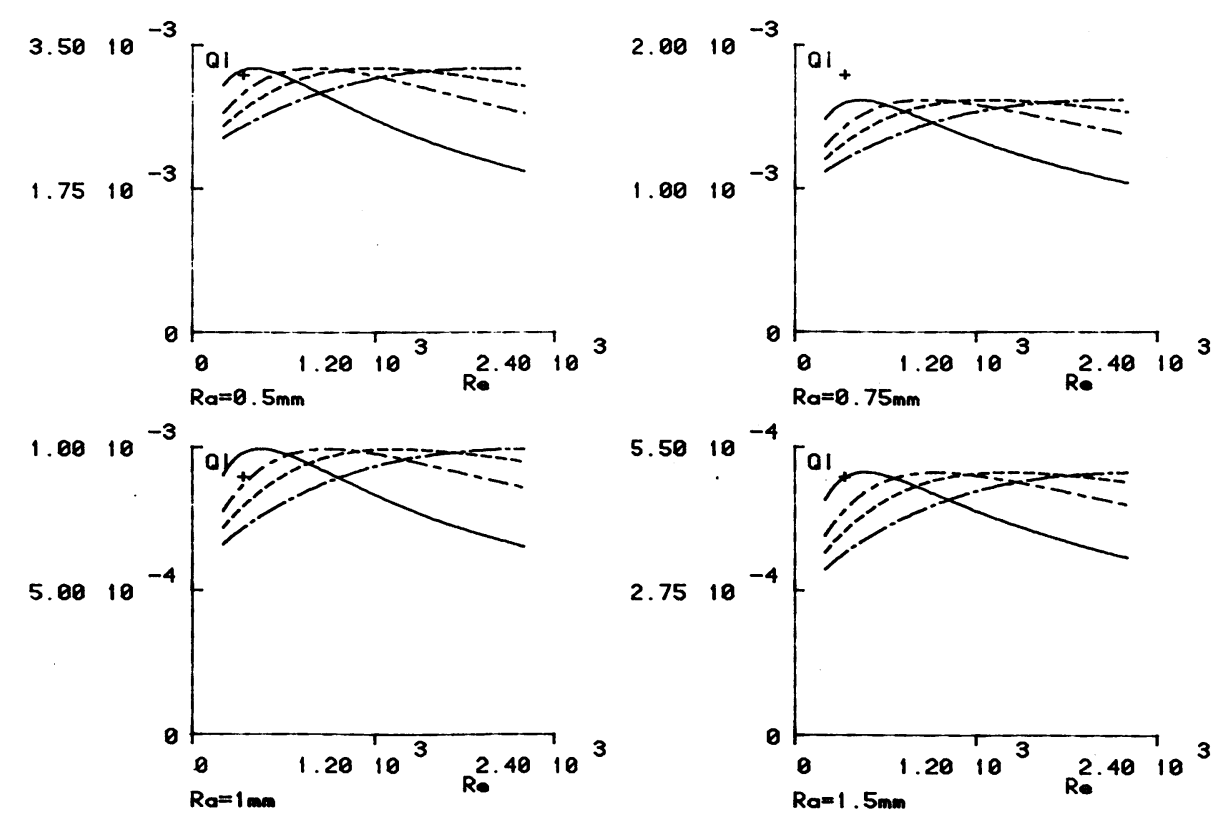

Fig. 4. - Evolution de la valeur théorique de la densité volumique de charge adimensionnelle convectée en fonction de l'épaisseur de l'orifice, de son diamètre et du nombre de Reynolds.

[Variation of the non-dimensionnal space charge density theoretically convected in terms of the orifice length and radius and the Reynolds number.] 
entre l'épaisseur de la couche diffuse et le rayon de l'orifice diminue, donc, pour que la charge décroisse en fonction du Reynolds, il faudrait que le profil de vitesse soit vraiment constant même dans la zone très proche de la paroi. Ce n'est pas le cas et c'est probablement pourquoi cette fois encore la charge convectée passe par un maximum en fonction du nombre de Reynolds. Afin de vérifier ce modèle, un montage expérimental, pour mesurer la charge convectée dans des orifices de différents diamètres et différentes longueurs, a été mis au point.

\section{Montage expérimental.}

Le montage utilisé est semblable à celui déjà utilisé pour mesurer des courants d'écoulements [12] et nous ne présenterons dans cette étude que ses spécificités résiduant principalement dans le remplacement du tube capillaire par un orifice circulaire (Fig. 5).

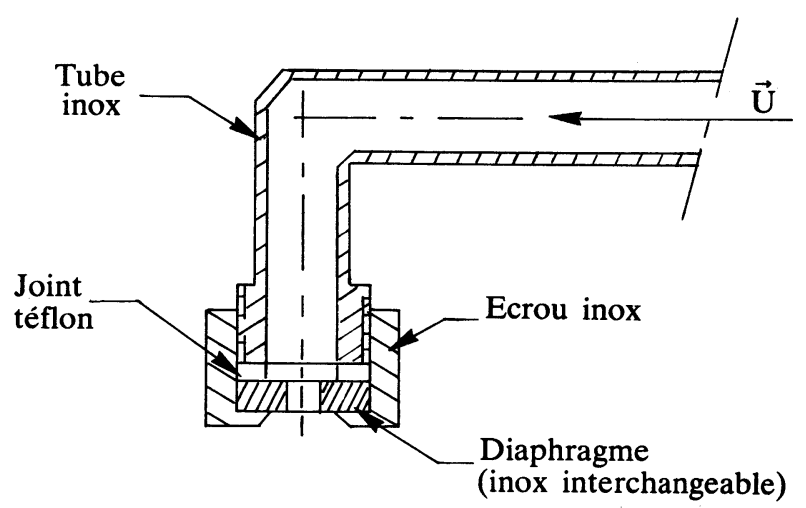

Fig. 5. - Schéma du support d'orifices.

[Diagram of the orifice support.]

\section{Résultats expérimentaux.}

Les mesures ont été faites pour de l'heptane technique de résistivité électrique égale à $0,87 \times 10^{11} \Omega \mathrm{m}$, pour 16 orifices d'épaisseur respective égale à $1 \mathrm{~mm}, 2 \mathrm{~mm}, 3 \mathrm{~mm}$ et $5 \mathrm{~mm}$, et de diamètre égaux à $1 \mathrm{~mm}, 1,5 \mathrm{~mm}, 2 \mathrm{~mm}$ et $3 \mathrm{~mm}$. La valeur de la densité volumique de charge convectée est représentée sur les figures $6,7,8$ et 9 . Nous voyons que l'allure de l'évolution de la densité volumique de charge convectée en fonction du nombre de Reynolds correspond assez bien aux prévisions théoriques, notamment elle passe par un maximum alors que l'hypothèse très simplifiée d'un profil plat dans toute la section aurait conduit à une décroissance de la charge.

Nous avons également représenté la charge convectée en fonction de la longueur des orifices pour $R e=2000$ et pour différents rayons (Fig. 10). On peut constater qu'elle est importante même pour de très petites épaisseurs.

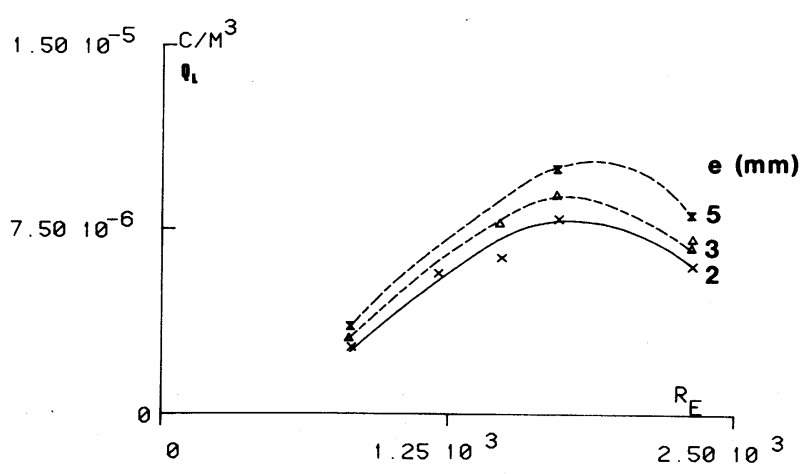

Fig. 6. - Charge transportée en fonction de $R e$ pour un orifice de $1 \mathrm{~mm}$ de diamètre.

[Charge convected in terms of $R e$ for an orifice of $1 \mathrm{~mm}$ of diameter.]

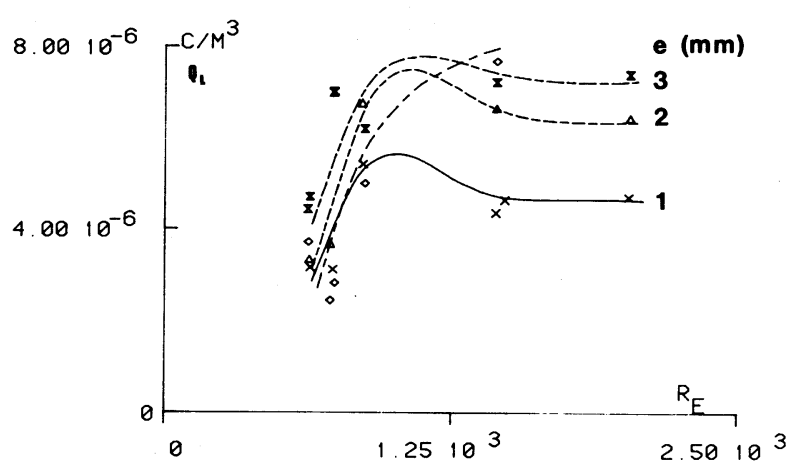

Fig. 7. - Charge transportée en fonction de $R e$ pour un orifice de $1,5 \mathrm{~mm}$ de diamètre.

[Charge convected in terms of $R e$ for an orifice of $1.5 \mathrm{~mm}$ of diameter.]

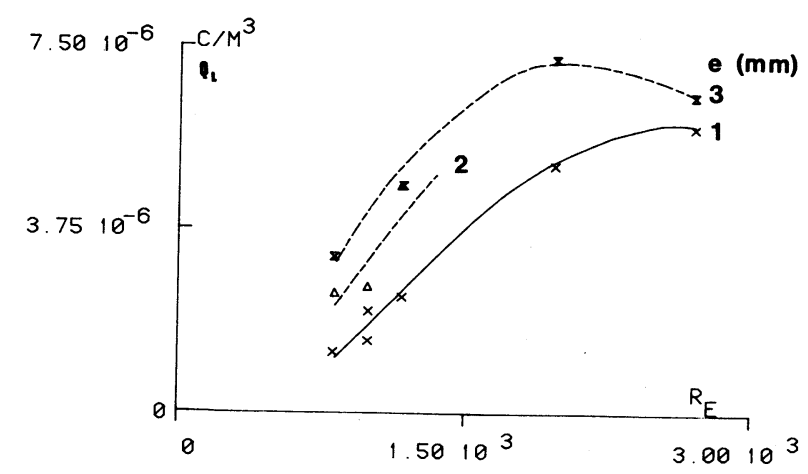

Fig. 8. - Charge transportée en fonction de $R e$ pour un orifice de $2 \mathrm{~mm}$ de diamètre.

[Charge convected in terms of $R e$ for an orifice of $2 \mathrm{~mm}$ of diameter.]

Sur la figure 11 , nous voyons l'évolution de la densité de charge à la paroi en fonction du temps de contact du liquide avec le métal. Cette courbe est très intéressante car elle montre que même pour des temps de contact très courts, la valeur de la charge à 


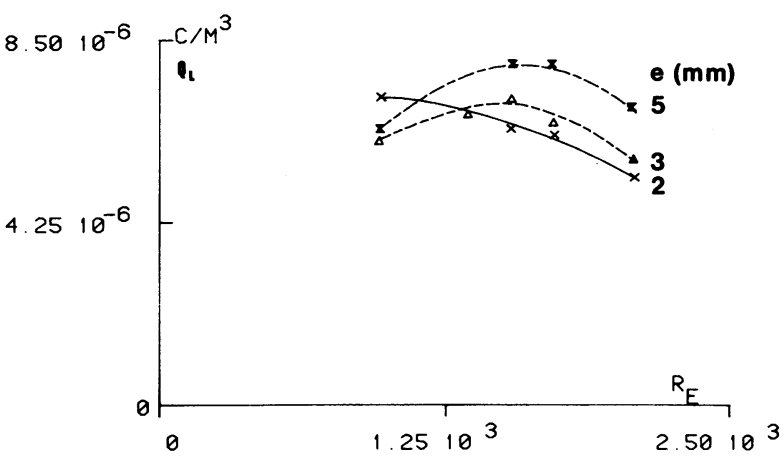

Fig. 9. - Charge transportée en fonction de $R e$ pour un orifice de $3 \mathrm{~mm}$ de diamètre.

[Charge convected in terms of $R e$ for an orifice of $3 \mathrm{~mm}$ of diameter.]

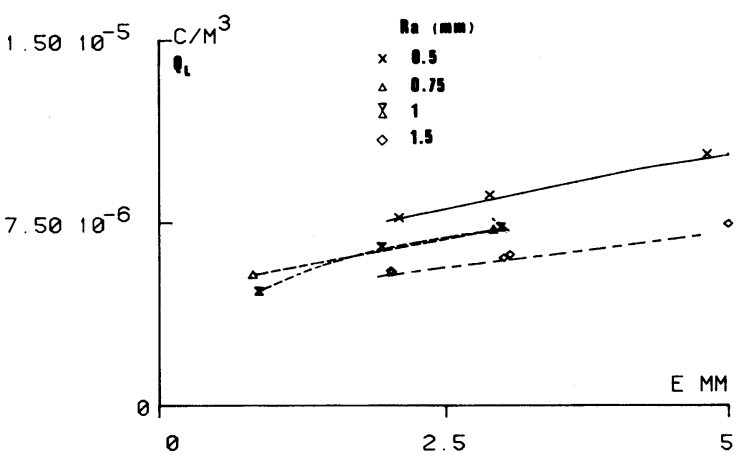

Fig. 10. - Charge transportée en fonction de la longueur du tube pour un nombre de Reynolds égal à 2000 .

[Charge convected in terms of the tube length for a Reynolds number equal to 2000 .]

la paroi est importante. Ainsi, le modèle choisi pour l'évolution de la densité volumique de charge en fonction de la distance à l'entrée du tube et du nombre de Reynolds $\left(\rho_{\mathrm{P}}=\rho_{\infty}(1-\exp (-b z))\right)$,

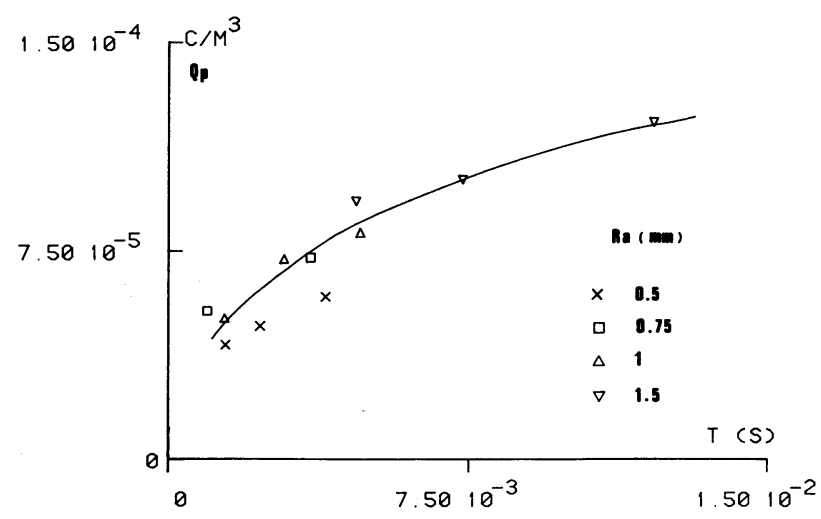

Fig. 11. - Charge à la paroi en fonction du temps de contact entre le liquide et la paroi de l'orifice.

[Wall charge in terms of contact duration between the liquid and the inner wall of the orifice.]

bien que convenant correctement pour des tubes plus longs, est erroné pour des orifices très courts, le courant de paroi dans les premières millisecondes après l'entrée du liquide étant bien plus important que celui existant ultérieurement. C'est ce courant tout au début du contact qui nous intéresse et que nous allons essayer de mesurer par la suite.

\section{Conclusion.}

Les mécanismes de génération de charge dans des orifices sont très intéressants car ils permettent de quantifier les phénomènes d'absorption aux premiers instants de contact entre le solide et le liquide. Les résultats obtenus nous montrent que la densité de courant semble très importante au tout début du contact.

Une étude récemmént entreprise, tenant compte du profil de vitesse exact et pour des orifices de longueur entre plus petite, doit nous permettre de mieux l'analyser.

\section{Bibliographie}

[1] Gosse, J. P., SolofomboAhANGy, A., J. Electrostatics 15 (1984).

[2] TouchaRD, G., J. Electrostatics (1978) 463-476.

[3] Touchard, G., Dumargue, P., J. Electrostatics 14 (1983) 209-223.

[4] Touchard, G., Romat, H., J. Electrostatics 10 (1981) 275-281.

[5] Sparrow, E. M., Lin, S. H., Lundgren, T. S., Phys. Fluids 7 (1964) 338-347.

[6] Aiello, G. A., Trefil, J. S., J. Biomech. 9 (1976) 49-54.
[7] TAO, L. N., GuPTA, S. D., 13th Middlewest Mechanics Conference.

[8] Romat, H., Thèse de $3^{\mathrm{e}}$ cycle, Poitiers, 1980.

[9] TouchaRD, G., 6th I.C.D.L., Rouen, 1978.

[10] Touchard, G., Romat, H., J. Electrostatics 12 (1982) 377-382.

[11] Touchard, G., Dumargue, P., Electrochim. Acta 20 (1975) 125-135.

[12] Touchard, G., Borzeix, J., Saunière, J., Rev. Gen. Electr. 7/8 (1985) 592-602. 